WASHINGTON, D.C.-The Biological Response Modifiers (BRM) Advisory Committee of the U.S. Food and Drug Administration (FDA, Bethesda, MD) has again shown that it will give a liberal reading to clinical data from relatively small trials-as long as the demonstrated risk from the drug under consideration is low.

The committee voted in December to recommend full FDA approval for two cytokines that combat infections by enhancing neutrophil counts-Immunex's (Seattle, WA) granulocyte macrophage-colony stimulating factor (GM-CSF, for use in conjunction with autologous bone marrow transplants [BMTs] and co-developed with Hoechst-Roussel Pharmaceuticals, Inc. [HRPI, Somerville, NJ]), and Amgen's (Thousand Oaks, CA) granulocyte-CSF (as an adjunct to chemotherapies treating non-myeloid malignancies). While the committee also supported a "conditional" approval for GM-CSF as a treatment for graft failure, the key to success before the advisory committee in both cases was a demonstration of enhanced patient quality of life during cancer chemotherapy. For the companies, the recommendations mean a shot at supplying the large, untapped market for white blood cell stimulating factors that conquer infections associated with cancer treatments.

For both drugs, approval turned on showing improvement in the quality of life of the treated cancer patients, rather than an absolute survival benefit. Relatively healthier patients are better able to withstand fulldose chemotherapy and are less likely to be pulled off a treatment regimen. Clinicians thus can use reductions in the number of culture-confirmed infections and use of intravenous antibiotics, as well as fewer days hospitalized versus the control groups, to demonstrate benefit.

One problem with studying GM\section{FIGHIING ABILITY}

CSF in this setting is that, in the words of Richard O'Reilly (Memorial Sloan-Kettering Cancer Center, New York), a consultant to the BRM Advisory Committee, "the disease overwhelms the data." A significant likelihood of relapse exists, and clinicians' inability to control the underlying cancers means that the trials are focused on "small points." Nevertheless, use of GM-CSF in transplant situations showed statistically significant declines in the number of serious infections, the level of neutropenia after a week of treatment, the amount of antibiotics used, and the number of days to discharge. While BRM Committee members did not unanimously embrace the use of absolute neutrophil count (ANC) as a correlate with the clinical effect of GM-CSF here, the data on improvements in the quality of life measures after ANC counts increased proved compelling.

The primary endpoint for G-CSF was defined more narrowly as a decline in the number of febrile infections, and the data showed a decline in the number of fever-associated infections as well as the level and duration of neutropenia. The clinicians also noted a trend towards improvement as measured by time on antibiotics and days in hospital, but the patient population measured was not large enough to be statistically relevant (due to the fact that many control patients "crossed over" to G-CSF early on).

What remained for the committee was to consider how broad an indication Amgen should receive: in terms of the kinds of chemotherapy regimens G-CSF could be used in conjunction with (the trials were based on use only with the CAE [cyclophosphamide/doxorubicin/etopocide] regimen) and the types of tumors to be treated. While the final vote to approve did include other regimens and

\section{RECOGNITED}

other solid tumors, committee members had some reservations. Fred Appelbaum (Fred Hutchinson Cancer Research Center, Seattle, WA) and others cautioned against using ANC as a surrogate measure in less aggressive chemotherapy regimens, and Paula Pitha (Johns Hopkins Hospital Oncology Center, Baltimore, MD) wanted data that first showed the growth hormone did not stimulate tumor growth in other cancers. That vote, on the types of cancers to treat, was the only one all session that did not carry unanimously.

The closest call for the Advisory Committee was in approving GMCSF therapeutically following graft failure. Immunex and HRPI had gone before the BRM Advisory Committee once before with their application (Bio/Technology 8:788, Sep. '90). But at that time, the committee was troubled by data ranging over trials at transplant centers that used varying initial patient selection criteria, preparative techniques, and strategies for timing of bone marrow transplants. It wanted to see a better indication of efficacy in a well-defined patient subgroup.

The committee expected that data on new patients would show a clearer trend, and Immunex and HRPI complied with its requests for a further analysis, including 66 new patients. But these new data still did not show, as committee member Michael Hawkins (National Cancer Institute, Bethesda, MD) observed, "a biological link" between ANC and the apparent clinical effect of GM-CSF. On the other hand, that effect, noted Hawkins and the others, "is real." And having just approved GM-CSF for autologous bone marrow transplants (and tacitly acknowledging that physicians will use GM-CSF "off-label" anyway), the committee had little real choice but to vote to approve.

-Mark Ratner 